\title{
Application of Absolute Alcohol in the Treatment of Traumatic Intracranial Hemorrhage via Interventional Embolization of Middle Meningeal Artery
}

\author{
Gangxian Fan, Henglu Wang, Jinke Ding, Chao Xu, Yongliang Liu, Chao Wang* and \\ Zefu $\mathrm{Li}^{*}$ \\ Department of Neurosurgery, Binzhou Medical University Hospital, Binzhou, China
}

OPEN ACCESS

Edited by:

Yunyan Wang,

Qilu Hospital, Shandong

University, China

Reviewed by:

Ulrika Sandvik,

Karolinska Institutet (KI), Sweden

Ralph George Depalma,

United States Department of Veterans

Affairs, United States

Jinlu Yu,

First Affiliated Hospital of Jilin

University, China

*Correspondence:

Chao Wang

chaowang_0628@163.com

Zefu Li

lizefu163@163.com

Specialty section:

This article was submitted to

Neurotrauma

a section of the journal

Frontiers in Neurology

Received: 22 December 2019

Accepted: 30 June 2020

Published: 06 August 2020

Citation:

Fan G, Wang H, Ding J, Xu C, Liu Y, Wang C and Li Z (2020) Application of Absolute Alcohol in the Treatment of Traumatic Intracranial Hemorrhage via Interventional Embolization of Middle

Meningeal Artery.

Front. Neurol. 11:824.

doi: 10.3389/fneur.2020.00824
Background: Traumatic brain injury is a common condition in neurosurgery. Traditional methods of treatment include conservative treatment and surgical evacuation using burr-holes or craniotomy; however, studies have reported problems such as high re-expansion rates after conservative treatment of epidural hematoma and high postoperative recurrence rates of subdural hematoma. Solutions to these problems are lacking, and research into new treatment methods is ongoing. Among the potential new treatments, middle meningeal arterial embolization is an option. This study involved patients with acute epidural hematoma and chronic subdural hematoma. The purpose was to evaluate the use and effects of absolute alcohol to embolize the middle meningeal artery to treat intracranial hematoma.

Material and Methods: A retrospective description study was 12 cases of intracranial hematoma who treated with absolute alcohol interventional therapy from our hospital between June 2018 and October 2019. Five patients with acute epidural hematoma and seven patients with chronic subdural hematoma were treated using absolute alcohol to embolize the middle meningeal artery. Patients' clinical data, imaging results, surgical results, and prognosis were recorded and analyzed.

Results: All patients underwent absolute alcohol embolization of the middle meningeal artery, in combination with burr-hole drainage. All imaging data were confirmed preoperatively. We successfully used absolute alcohol to embolize the middle meningeal artery intraoperatively and confirmed these results by postoperative angiography. All patients achieved symptomatic relief without complications, and no recurrence or re-expansion was seen with follow-up computed tomography. Our study has been registered in the Chinese Clinical Trial Registry (http://www.chictr.org. cn, ChiCTR1800018714).

Conclusion: The use of absolute alcohol to embolize the middle meningeal artery could be used as an attempt to treat acute epidural hematoma and chronic subdural hematoma.

Keywords: absolute alcohol, embolization, acute epidural hematoma, chronic subdural hematoma, middle meningeal artery 


\section{INTRODUCTION}

Traumatic brain injury is a common neurosurgical condition, and it often causes traumatic intracranial hemorrhage (TICH) (1). The TICH affects over 10 million people annually leading to either mortality or hospitalization (2). Subdural hemorrhage, epidural hemorrhage and intraparenchymal hemorrhage make up most TICHs (3). Patients with TICH frequently undergo neurologic deterioration due to an expanding hematoma. Even with surgical intervention, such patients experienced poor longterm outcomes (4).

Acute epidural hematoma (AEDH) is one of the most serious TICH and is often accompanied by sudden neurological symptoms (5). The most common cause is trauma leading to rupture of the middle meningeal artery (MMA), and progression is relatively rapid, sometimes requiring emergency surgery (6). Chronic subdural hematoma (CSDH) is a frequent TICH with incidence rates varying from 5.3 to 13.5 cases per 100,000 persons/year in the general population with a higher incidence among older adults (7). Surgical treatment is the first choice for TICH and consists of surgical evacuation using burr-holes or craniotomy $(6,8,9)$. However, several studies reported a rate of re-expansion of acute small epidural hematomas after conservative treatment of 23 to $65 \%(10,11)$, and some studies report a postoperative recurrence rate for $\mathrm{CSDH}$ of 0.35 to $23 \%$ or higher $(12,13)$. Furthermore, previous studies have found that there was a link between the expansion of AEDHs and the recurrence of CSDHs with the MMA. In order to prevent the re-expansion and recurrence of hematomas, they tried to use a minimally invasive method, MMA embolization in combination with burr-hole drainage (14-16). Currently, many materials were reported to achieve MMA embolization, and the embolization materials could be solid (polyvinyl alcohol particles, coils, and gelatin sponges) or liquid (n-butyl-2-cyanoacrylate) $(17,18)$. Absolute alcohol is the liquid sclerosing agent most often used because of its low cost, wide availability, and ease of use. It can destroy endothelial cells, and induce thrombosis by denaturing blood proteins, denuding the vascular wall of endothelial cells and precipitating their protoplasm (19-22). Alcohol has been widely-used in endovascular therapy to treat arteriovenous malformations, hemangioma, arteriovenous fistula, and other diseases, and is a popular material because of its low cost and efficacy $(16,22)$.

Although some studies report MMA embolization to treat intracranial hematoma, none, to our knowledge, have reported using absolute alcohol for this treatment. In this study, we report the successful use of MMA embolization with absolute alcohol to treat intracranial hematoma in 12 patients: five patients with $\mathrm{AEDH}$ and seven patients with CSDH.

\section{MATERIALS AND METHODS}

\section{Patient Selection}

We retrospectively analyzed data for all patients with intracranial hematomas treated at our center between June 2018 and October 2019. Inclusion criteria of patients were: [1] all patients were clearly diagnosed as AEDH or CSDH in brain CT;
[2] all patients' midline shift of the brain CT was $<10 \mathrm{~mm}$; [3] absolute alcohol was used in the MMA embolization. We excluded patients who underwent MMA embolization with other materials or new onset of dangerous anastomosis during intraoperative cerebral angiography and other underlying conditions (vascular lesions, brain tumor, arachnoid cyst, or previous craniotomy).

In the retrospective arm of the study, using the clinical data warehouse system of our institution, we reviewed 68 patients with $\mathrm{AEDH}$ or $\mathrm{CSDH}$ who were given a diagnosis, and 56 patients were excluded: 36 with AEDH or CSDH used other materials for MMA embolization; six with space occupying lesion (arachnoid cyst, intracranial tumor, et al.); five with urokinase injection; four with after craniotomy; three with vascular lesions (arteriovenous malformation, moyamoya disease, et al.), and two with dangerous anastomosis. Finally, according to our general neurosurgical diagnosis and treatment procedures, 12 patients were enrolled. A standard operative protocol was followed in all patients. We recorded patients' demographic information, clinical presentation, procedural methods, computed tomographic (CT) characteristics, angiographic changes, management, and outcomes. This study was carried out in accordance with the recommendations of the neurosurgical diagnosis and treatment guidelines, the ethic committee of Binzhou medical university hospital. The protocol was approved by our institutional ethics committee (No. 2018065). All subjects gave written informed consent in accordance with the Declaration of Helsinki.

\section{MMA Embolization}

We performed MMA embolization under local anesthesia. First, the femoral artery was punctured using Seldinger's technique, and passage through the femoral artery was obtained using a standard 6-French sheath. This procedure was followed by external carotid arterial angiography, placing an Excelsior ${ }^{\circledR}$ SL-10 microcatheter (Target Therapeutics/Boston Scientific, Fremont, CA, USA) under fluoroscopic guidance and placing a Traxcess ${ }^{\circledR} 14$ microguidewire (MicroVention, Tustin, CA, USA) into the trunk of middle meningeal artery followed by slow injection of absolute alcohol through the microcatheter for embolization $(23,24)$. Based on previous reports and our experience using absolute alcohol in our institution (25, 26), we mixed alcohol and contrast agent in an 8:2 ratios and used a dose of $0.03 \mathrm{ml} / \mathrm{kg}$ mixture for embolization, which we injected slowly. After injection, we removed the syringe and evaluated the extent of embolization. Also after each alcohol injection, and before removing the syringe, we waited for $\sim 5 \mathrm{~min}$ to allow for complete action of the alcohol. Next, we removed the guiding catheter and the femoral arterial sheath and placed an occlusion in the femoral arterial puncture site. Generally, we treated each patient's hematoma condition in combination with burr-hole drainage to relieve their symptoms, improve prognosis, and reduce the recurrence rate or re-expansion rate. Postoperatively, all patients were transferred to the neurosurgical intensive care unit or ward for observation. 

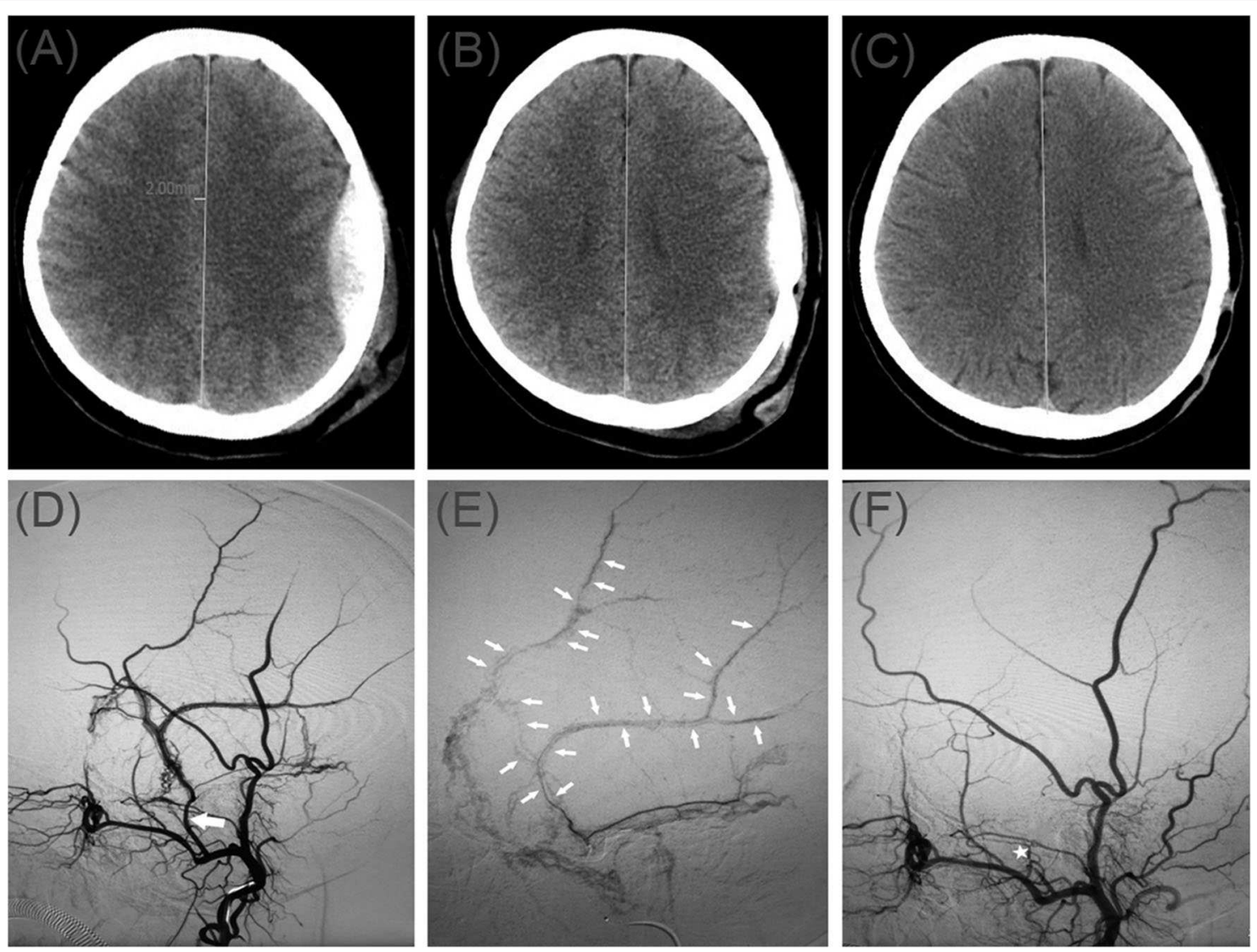

FIGURE 1 | (A) Brain computed tomographic hematoma images for patient 1 before treatment (midline shift: $2.00 \mathrm{~mm}$ ). (B) Brain CT was repeated on postoperative day 11. (C) Follow-up brain CT was performed at 88 days. (D) Pre-operative DSA presented abnormal MMA (Large arrow). (E) Intra-operative DSA showed contrast agent extravasation or arteriovenous fistula (Small arrow). (F) Postoperative DSA found that the MMA disappeared (Asterisk). DSA, digital subtraction angiographic.

\section{Follow-Up}

Hematomas in all patients were diagnosed or identified by CT scan on admission, and imaging was performed once per week or according to changes of the clinical manifestations in individual patients during hospitalization. All patients were discharged once their symptoms improved. A monthly repeat brain CT was recommended, and we followed all patients for 4-6 months after discharge. We readmitted patients if symptoms reappeared or worsened.

\section{RESULTS}

Table 1 shows the summary of the 12 cases. Ten patients were men, two were women, and their mean age was 54.2 years (range, 18-78 years). Ten patients had a history of head trauma. The Glasgow Coma Scale (GCS) on admission was higher than 13 in all patients. Among all patients, the most common clinical symptoms were headache, dizziness, drowsiness, vomiting, hemiparesis. All imaging data were confirmed preoperatively. All patients underwent absolute alcohol embolization of the middle meningeal artery, in combination with burr-hole drainage.
In all patients, embolization was successful, without complications, and bleeding was stopped immediately. There was no evidence of recurrent bleeding after the follow-up brain $\mathrm{CT}$, and the hematoma cavity gradually decreased in size, thereafter. CSDHs may recur and AEDHs may expand after follow-up. However, these conditions were not identified during the follow-up period.

\section{Representative Cases' Clinical Data Patient 3}

A 50-year-old women injured in a traffic accident and was admitted to the hospital. His Glasgow Coma Scale score was 14, and clinical manifestations were mainly headache and dizziness. A CT scan disclosed a small EDH on the left side (Figure 1A). His symptoms changed on subsequent examinations, and a new symptom of vomiting appeared. In order to relieve the symptoms and prevent the enlargement of the hematoma, we took surgical treatment. The interval time between the injury and surgery was $<2 \mathrm{~h}$. First, he underwent MMA embolization. And subsequent burr-hole drainage was taken under local anesthesia. We performed cerebral angiography pre-, intra-, and postoperatively 
TABLE 1 | Demographic data, clinical and imaging findings of the patients.

\begin{tabular}{|c|c|c|c|}
\hline Characteristics & & $\begin{array}{c}\text { Acute epidural } \\
\text { hematoma }(N=5)\end{array}$ & $\begin{array}{l}\text { Chronic subdural } \\
\text { hematoma }(N=7)\end{array}$ \\
\hline \multirow[t]{3}{*}{ Age } & $\leq 30$ & 1 & 1 \\
\hline & $\begin{array}{l}30< \\
\leq 60\end{array}$ & 3 & 2 \\
\hline & $>60$ & 1 & 4 \\
\hline \multirow[t]{5}{*}{ clinical symptoms ${ }^{a}$} & Headache & 2 & 5 \\
\hline & Dizziness & 2 & 2 \\
\hline & Drowsiness & 1 & 1 \\
\hline & Vomiting & 2 & 1 \\
\hline & hemiparesis & 1 & 2 \\
\hline \multirow[t]{2}{*}{ GCS $^{b}$ score } & 14 & 3 & 4 \\
\hline & 15 & 2 & 3 \\
\hline \multirow[t]{4}{*}{$\begin{array}{l}\text { The size of } \\
\text { hematoma (ml) }\end{array}$} & $\begin{array}{l}10<, \\
\leq 15\end{array}$ & 2 & 0 \\
\hline & $\begin{array}{l}15< \\
\leq 20\end{array}$ & 3 & 1 \\
\hline & $\begin{array}{l}20<, \\
\leq 25\end{array}$ & 0 & 2 \\
\hline & $\begin{array}{l}25< \\
\leq 30\end{array}$ & 0 & 4 \\
\hline \multirow[t]{2}{*}{ Hematoma side } & Left & 2 & 4 \\
\hline & Right & 3 & 3 \\
\hline
\end{tabular}

a Clinical symptoms data was a summary of symptoms rather than the number of patients; b GCS: Glasgow Coma Scale.

for MMA embolization (Figures 1D-F). His symptoms improved, and there were no signs of re-expansion or recurrence of the hematoma on CT brain scan before discharge. The hematoma cavity gradually decreased in size, thereafter (Figures 1B,C).

\section{Patient 4}

A 49-year-old man injured in an accidental fall and was admitted to the emergency room without neurological abnormalities. His Glasgow Coma Scale score was 14. A CT scan disclosed a AEDH on the left side (Figure 2A). On a subsequent neurological examination, his score on the Glasgow Coma Scale was 15. However, the headache symptoms of the patients were more obvious. In order to relieve the symptoms and prevent the enlargement of the hematoma, we took surgical treatment. The interval time between the injury and surgery was $<2 \mathrm{~h}$. First, he underwent MMA embolization. And subsequent burr-hole drainage was taken under local anesthesia. We performed cerebral angiography pre-, intra-, and postoperatively for MMA embolization (Figures 2D-F). His symptoms improved, and there were no signs of re-expansion or recurrence of the hematoma on CT brain scan before discharge. The hematoma cavity gradually decreased in size, thereafter (Figures 2B,C).

\section{Patient 7}

A 70-year-old man was admitted to our hospital for weakness in his left limb. He had a head injury 3 months ago. His
Glasgow Coma Scale score was 14. A CT scan disclosed multiple site $\mathrm{SDH}$ on the right side (Figure 3A). His GCS score improved on subsequent examination, but his left lower limb was gradually weak, which presented only four levels of muscle strength. In order to relieve symptoms and recurrence of hematoma, we took surgical treatment. First, he underwent MMA embolization and burr-hole drainage under local anesthesia. We performed cerebral angiography pre-, intra, and postoperatively for MMA embolization (Figures 3D-F). No imaging of the middle meningeal artery was observed in postoperative angiography, and postoperatively, his symptoms improved. There was no evidence of rebleeding after repeat brain CT before discharge, and the hematoma cavity gradually decreased in size, thereafter (Figures 3B,C).

\section{Patient 9}

A 78-year-old man was admitted to our hospital with a duration of sudden-onset headache. He had a head injury 2 months ago. His Glasgow Coma Scale score was 14. A CT scan disclosed multiple site SDH on the left side (Figure 4A). On a subsequent neurological examination, his symptoms was more serious in headache and a new symptom of vomiting appeared. First, he underwent MMA embolization and burr-hole drainage under local anesthesia. We performed cerebral angiography pre-, intra-, and postoperatively for MMA embolization (Figures 4D-F). No imaging of the middle meningeal artery was observed during postoperative angiography, and postoperatively, his symptoms improved. There was no evidence of recurrent bleeding after repeat brain CT before discharge, and the hematoma cavity gradually decreased in size, thereafter (Figures 4B,C).

\section{DISCUSSION}

In our study, we treated 12 patients with $\mathrm{TICH}$ who underwent minimally-invasive treatment with absolute alcohol embolization of the MMA. According to their symptoms and imaging findings, all patients underwent MMA embolization in combination with burr-hole drainage under local anesthesia. The purpose of embolization was to treat the bleeding source and reduce the re-expansion or recurrence of hematoma, and the purpose of burr-hole drainage was to alleviate patients' symptoms. In our study, all patients' symptoms alleviated postoperatively, and patients were discharged after symptom improvement. Based on brain CT findings, no patients experienced hematoma recurrence after discharge, and the hematoma cavity decreased significantly in size over time.

\section{Mechanisms for the Development of AEDH and CSDH, and Related Digital Subtraction Angiography (DSA) Findings}

$\mathrm{AEDH}$ is a rapid-onset and severe traumatic brain injury (5). Arterial bleeding is responsible for $85 \%$ hematomas in AEDHs. Among them, the middle meningeal artery (MMA) is the most common source of EDHs $(10,27)$. To begin with, 

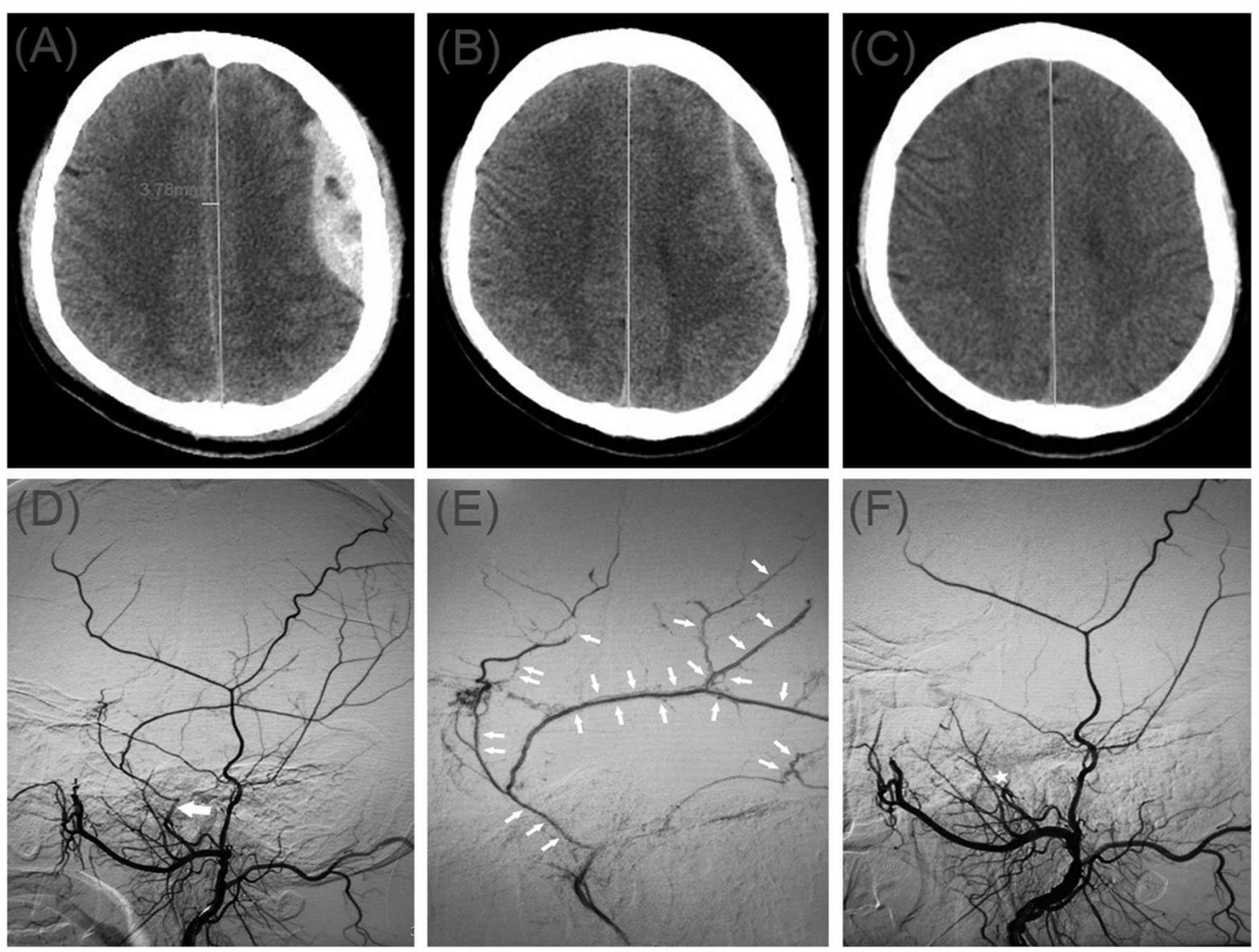

FIGURE 2 | (A) Brain computed tomographic hematoma images for patient 2 before treatment (midline shift: 3.78 mm). (B) Brain CT was repeated on postoperative day 10. (C) Follow-up brain CT was performed at 67 days. (D) Pre-operative DSA presented abnormal MMA (Large arrow). (E) Intra-operative DSA showed contrast agent extravasation or arteriovenous fistula (Small arrow). (F) Postoperative DSA found that the MMA disappeared (Asterisk).

small EDHs are usually treated conservatively. Nevertheless, in some large samples of EDHs, it was found that the rate of re-expansion of hematoma after conservative treatment reached $5.5 \sim 65 \%(9,28)$. Sakai et al. showed that $65 \%$ of small EDHs re-expansion in the first $24 \mathrm{~h}$ after trauma (29). A prospective study evaluating 22 patients who had small EDHs showed that $32 \%$ of the patients underwent surgical evacuation because of late enlargement (30). Furthermore, most studies of EDHs suggest that EDHs are related to tearing or damage to the MMA, seen as contrast agent extravasation, pseudoaneurysm, or arteriovenous fistula in DSA $(28,31)$. However, the best treatment option for EDH in larger or occipital and non-middle meningeal arterial blood supply areas remains surgery (32).

$\mathrm{CSDH}$ is being diagnosed more commonly in neurosurgical practice, and burr-hole drainage is the gold standard for patients with space-occupying $\mathrm{CSDH}$ (12). One hypothesis for the cause of $\mathrm{CSDH}$ is tearing of one or more bridging veins, which causes bleeding into the dural border cell layer and leads to $\mathrm{CSDH}$ enlargement $(7,8)$. However, evidence currently indicates that the responsible blood vessels for CSDH may arise from the MMA. Takizawa et al. observed that the MMA was enlarged with CSDH (33). Several studies have shown that selective MMA angiography revealed diffuse dilatation of the MMA and visualization of scattered abnormal vascular networks reflecting macrocapillaries, a sinusoidal channel layer, and giant capillaries in the outer membrane $(13,34)$. Additionally, a "cotton wool-like staining" appearance of the distal MMA vasculature and "wispiness" (or the neovasculature) of the distal MMA branches may be observed in DSA (35).

\section{Treatment for AEDH and CSDH}

MMA embolization has been reported for the treatment of EDH $(11,36)$. The first published series of the endovascular treatment of AEDH was described by Suzuki et al. (6), and the authors reported successful embolization in nine patients with EDHs and associated lesions. Zhang et al. reported a novel minimally-invasive method of endovascular embolization under local anesthesia to treat $\mathrm{AEDH}$, which is especially useful for older patients or patients unable to undergo general anesthesia (10). Furthermore, to date, the use of urokinase with clot aspiration has emerged as the most promising surgical modality for intracerebral hemorrhage and intra-ventricular 

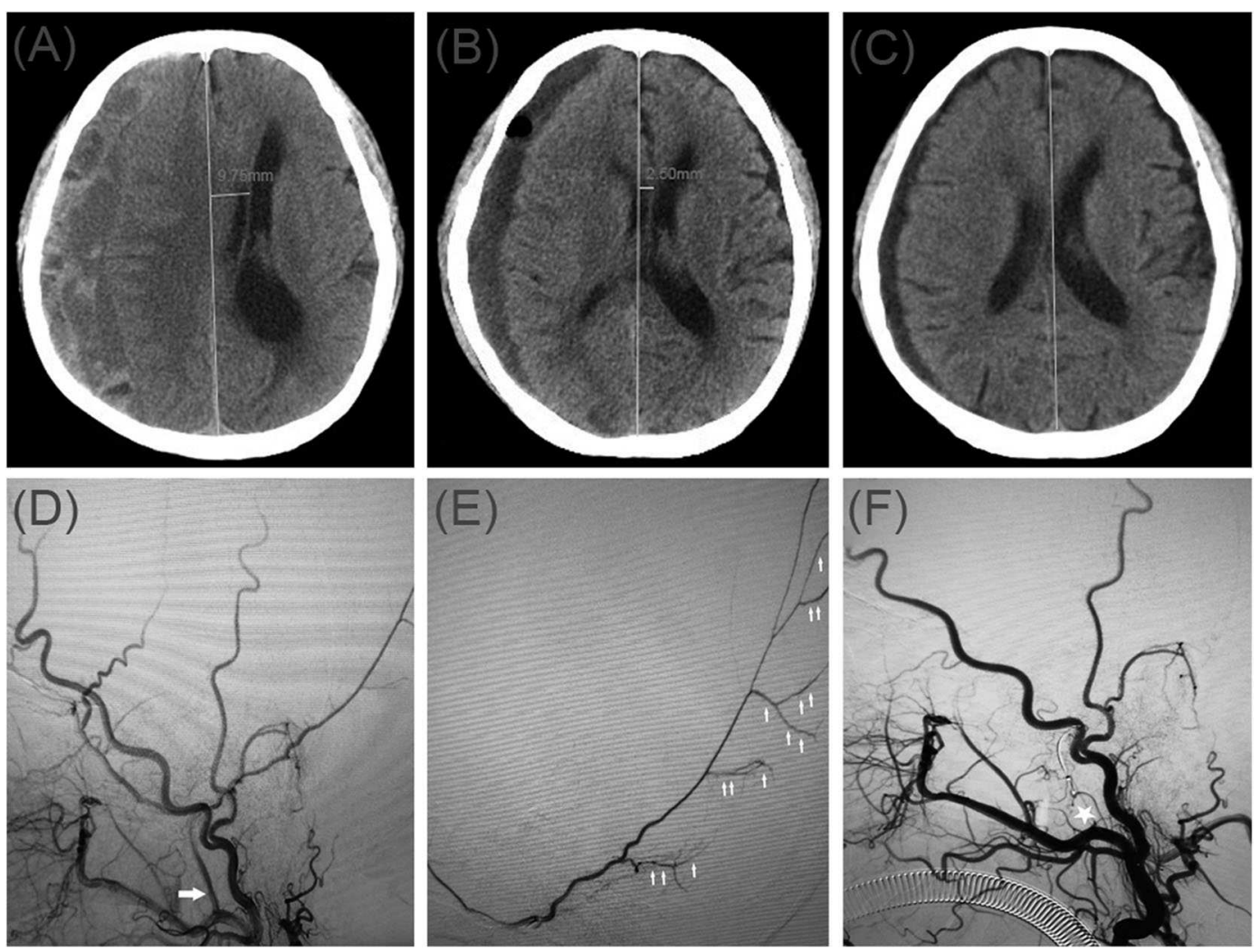

FIGURE 3 | (A) Brain computed tomographic hematoma images for patient 3 before treatment (midline shift: 9.75 mm). (B) Brain CT was repeated on postoperative day 12 (midline shift: 2.50 mm). (C) Follow-up brain CT was performed at 58 days. (D) Pre-operative DSA presented abnormal MMA (Large arrow). (E) Intra-operative DSA showed contrast agent extravasation or cotton wool-like staining (Small arrow). (F) Postoperative DSA found that the MMA disappeared (Asterisk).

hemorrhage $(5,37,38)$. According to this fact, some researchers resorted to urokinase to facilitate the liquifaction of lots for aspiration of the AEDH. However, early application of urokinase may have a certain risk of bleeding, which is generally used after operation $(10,38,39)$. This purpose can effectively dissolve the hematoma without increasing the risk of longterm bleeding. The urokinase $(20 \mathrm{ku}$ resolved in $3-\mathrm{mL}$ saline, twice a day) was injected into the hematoma cyst by drainage tube to lyse hematoma on the first postoperative day. The reason why our patients did not use urokinase was that our patients had a relatively small amount of hematoma, MMA embolization and burr-hole drainage without urokinase was enough for preventing hematoma expansion and removing the occupying effect of hematoma. Peres et al. and Ross et al. demonstrated that MMA embolization was highly effective and safe to achieve size stabilization in non-surgically-treated $\operatorname{AEDH}(28,40)$.

Treatment for CSDH should address the capillary feeders of the hematomas that originate from the MMA. Hashimoto et al. found that MMA embolization was effective for refractory $\mathrm{CSDH}$ or $\mathrm{CSDH}$ patients with a risk of recurrence, and this method is considered effective to stop hematoma enlargement and promote resolution (41). Link et al. and Kim et al. reported that MMA embolization was a minimally-invasive and low-risk initial alternative to surgery for symptomatic CSDH when clinically appropriate $(13,17)$.

According to the above related reports, our research has tried to use absolute alcohol to embolize the middle meningeal artery to treat $\mathrm{AEDH}$ or $\mathrm{CSDH}$, and observed that absolute alcohol was feasible as an embolization agent for middle meningeal artery embolization.

\section{Important Considerations in MMA Embolization}

MMA embolization is a rapid technique, but a complete angiographic examination should be performed before embolization. To begin with, Studies have shown that anastomotic branches can be present between the externaland the internal carotid arteries, and inappropriate embolization of these anastomotic branches can cause serious complications; sometimes the central retinal artery originates from the MMA (10, 30). Intraoperatively, surgeons should check for an anastomosis between the MMA and the ophthalmic artery. If a dangerous anastomosis is observed, embolization injection should be performed carefully. Studies have reported the presence of extracranial-intracranial anastomoses of the external carotid artery with an average diameter of $>100 \mathrm{um}$ 

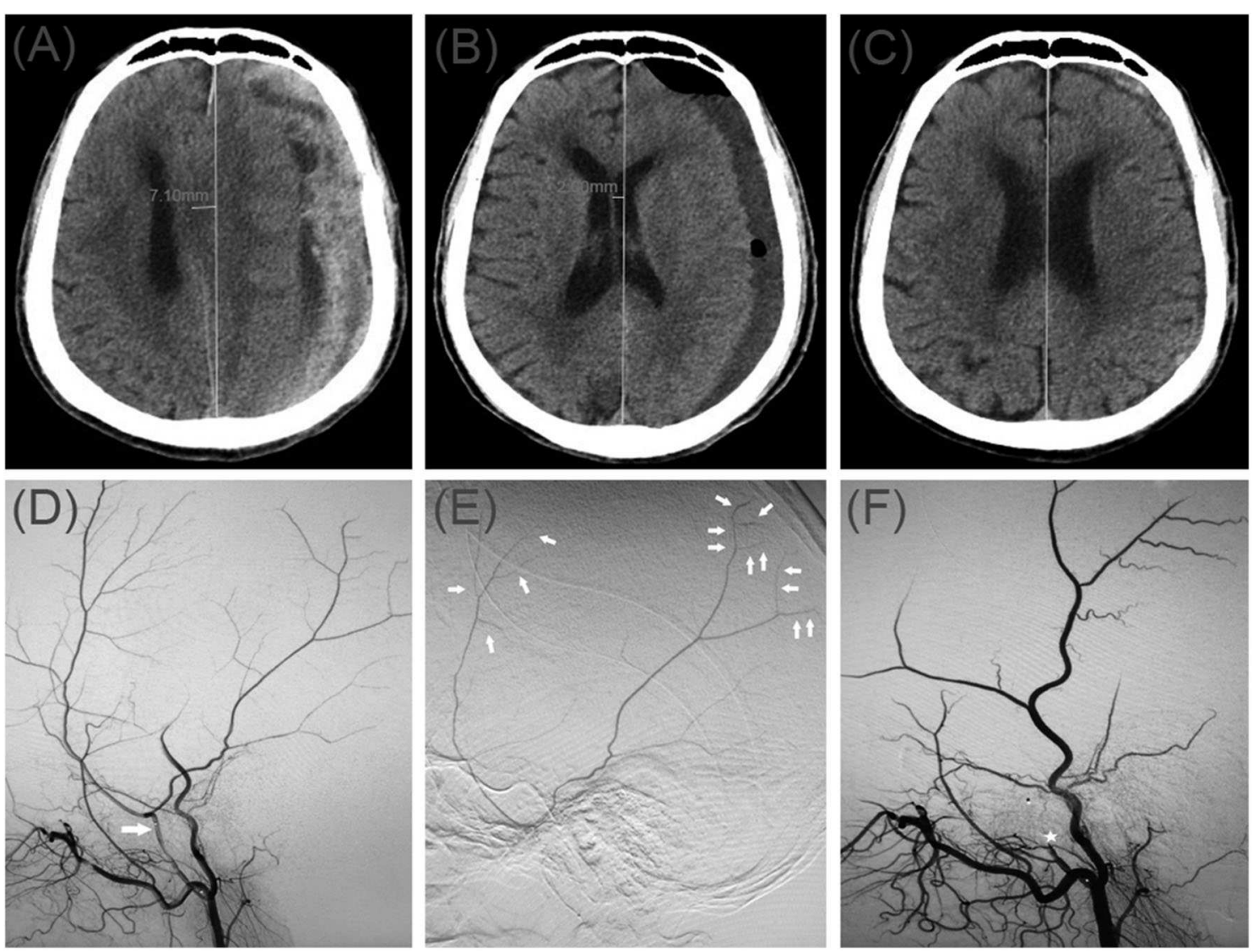

FIGURE 4 | (A) Brain computed tomographic hematoma images for patient 4 before treatment (midline shift: $7.10 \mathrm{~mm}$ ). (B) Brain CT was repeated on postoperative day 14 (midline shift: 2.00 mm). (C) Follow-up brain CT was performed at 72 days. (D) Pre-operative DSA presented abnormal MMA (Large arrow). (E) Intra-operative DSA showed contrast agent extravasation or cotton wool-like staining (Small arrow). (F) Postoperative DSA found that the MMA disappeared (Asterisk).

(10, 28, 42). Generally, previous studies used embolization materials with a diameter much larger than the diameter of the anastomoses; therefore, no complications were seen related to the embolization material entering the intracranial blood vessels through the anastomosis and leading to cerebral infarction or cranial nerve dysfunction. In our study, we excluded patients with a dangerous anastomosis, thus we did not observe a complication due to inappropriate embolization during our follow-up. Furthermore, there was currently no agreement on the site of MMA embolism: trunk or distal $(11,17,18)$. Link et al. suggested that during embolization, physicians should proceed carefully to avoid inappropriately embolizing the petrosal branch to avoid facial nerve dysfunction (35). However, other studies showed that the petrosal branch was not the only blood supply to the facial nerve (43). This may be the reason why we embolized the trunk of MMA without new symptoms.

\section{Use of Alcohol in Endovascular Interventional Therapy}

Alcohol has been widely-used in endovascular therapy because of its low cost and ease of access. Alcohol is effective in the intravascular treatment of vascular malformations, dysfunctions, and hematomas $(14,22)$. Alcohol may act by promoting coagulation of proteins in the blood or lymphatic fluid and destroying the cellular lining of the abnormal vessel (26). Singh et al. found that absolute alcohol embolization was safe and effective to treat vertebral hemangiomas with severe spinal cord compression (24). Additionally, Chen et al. demonstrated that using absolute alcohol for percutaneous embolization to treat venous malformations was safe and effective (15). The volume of alcohol injected is based on the size and location of the malformation and the patient's weight (44), and injection is stopped when resistance to manual injection increases or when alcohol is visualized exiting the lesion in the venous drainage (45). The maximum recommended and safe dose of alcohol is $1 \mathrm{ml} / \mathrm{kg}$ body weight and can be used with proper precautions $(21,44,45)$. Based on previous reports and our experience, we explored an appropriate amount of alcohol for embolization of the blood vessels responsible for intracranial hematoma.

The results of this study are promising, but our conclusions are limited. The number of patients in this study was small, and the study lacked a control group. We also did not compare 
our procedure with previous treatments or embolization with other embolic materials. We also had no standard way to measure outcomes; therefore, there may be limitations related to our measurements. Prospective randomized case-control studies are needed to evaluate the efficacy of absolute alcohol embolization, and we expect that not all patients will be treated successfully using this technique. Despite the limitations, embolization provided a possible new method of MMA embolization in younger patients or those intolerant to general anesthesia. Absolute alcohol MMA embolization could also become a new, low-cost treatment for refractory problems. Our approach could make minimally-invasive MMA embolization more available.

\section{CONCLUSIONS}

The effect and prognosis in patients receiving absolute alcohol MMA embolization in our study were similar to results in studies using other embolic materials. Because of its low cost and ease of access, it may be used as a recommended MMA embolization material in the future when treating $\mathrm{AEDH}$ and $\mathrm{CSDH}$.

\section{DATA AVAILABILITY STATEMENT}

The datasets generated for this study are available on request to the corresponding author.

\section{REFERENCES}

1. Hyder A, Wunderlich C, Puvanachandra P, Gururaj G, Kobusingye $\mathrm{O}$. The impact of traumatic brain injuries : a global perspective. NeuroRehabilitation. (2007) 22:341-53. doi: 10.3233/NRE-2007-22502

2. Dunham C, Hoffman D, Huang G, Omert L, Gemmel D, Merrell R. Traumatic intracranial hemorrhage correlates with preinjury brain atrophy, but not with antithrombotic agent use : a retrospective study. PLoS ONE. (2014) 9:e109473. doi: 10.1371/journal.pone.0109473

3. Lan M, Dambrino R, Youssef A, Yengo-kahn A, Dewan M, Ehrenfeld J, et al. Repeat surgery after decompressive craniectomy for traumatic intracranial hemorrhage: outcomes and predictors. World Neurosurg. (2020) 133:e75766. doi: 10.1016/j.wneu.2019.09.148

4. Van Ornam J, Pruitt P, Borczuk P. Is repeat head CT necessary in patients with mild traumatic intracranial hemorrhage. Am J Emerg Med. (2019) 37:1694-8. doi: 10.1016/j.ajem.2018.12.012

5. Bullock M, Chesnut R, Ghajar J, Gordon D, Hartl R, Newell $\mathrm{D}$, et al. Surgical management of acute epidural hematomas. Neurosurgery. (2006) 58:7-15. doi: 10.1227/01.NEU.0000210363.9 1172.A8

6. Suzuki S, Endo M, Kurata A, Ohmomo T, Oka H, Kitahara T, et al. Efficacy of endovascular surgery for the treatment of acute epidural hematomas. Am J Neuroradiol. (2004) 25:1177-80. Available online at: http://www.ajnr. org/content/25/7/1177

7. Andersen-Ranberg NC, Poulsen FR, Bergholt B, Hundsholt T, Fugleholm K. Bilateral chronic subdural hematoma: unilateral or bilateral drainage? J Neurosurg. (2017) 126:1905-11. doi: 10.3171/2016.4.JNS1 52642

8. Almenawer SA, Farrokhyar F, Hong C, Alhazzani W, Manoranjan B, Yarascavitch B, et al. Chronic subdural hematoma management: a systematic

\section{ETHICS STATEMENT}

Written informed consent was obtained from the individuals for the publication of any potentially identifiable images or data included in this article.

\section{AUTHOR CONTRIBUTIONS}

GF drafted the manuscript. CW and CX completed the operation. HW, JD, and YL performed the data collection and data analysis. CW and ZL participated in the design of this study and helped to check the manuscript. All authors read and approved the final manuscript. All authors contributed to the article and approved the submitted version.

\section{FUNDING}

This work was supported by Shandong Province Natural Science Foundation of China (ZR2017LH033, ZR2018LH007) and Binzhou Medical University Scientific Research Foundation (BY2016KYQD15, BY2018KJ02).

\section{ACKNOWLEDGMENTS}

We acknowledge assistance from the endovascular Neurosurgery Research Group members (Department of Neurosurgery, Binzhou Medical University Hospital, Binzhou, Shandong, China). review and meta-analysis of 34829 patients. Ann Surg. (2014) 259:44957. doi: 10.1097/SLA.0000000000000255

9. De-Andrade AF, Figueiredo EG, Caldas JG, Paiva WS, De Amorim RL., Puglia $P$, et al. Intracranial vascular lesions associated with small epidural hematomas. Neurosurgery. (2008) 62:416-21. doi: 10.1227/01.neu.0000316008.11388.f2

10. Zhang Y, Li Q, Zhao R, Yang Z, Li Y, Min W, et al. Novel minimally invasive treatment strategy for acute traumatic epidural hematoma: endovascular embolization combined with drainage surgery and use of urokinase. World Neurosurg. (2018) 110:206-9. doi: 10.1016/j.wneu.2017.11.047

11. Kim DH, Lee JY, Jeon HJ, Cho BM, Park S-H, Oh S-M. Intraoperative endovascular embolization of middle meningeal artery and a pseudoaneurysm by using N-Butyl 2-cyanoacrylate for hemostasis during operation of acute epidural hemorrhage. Korean J Neurotrauma. (2015) 11:167. doi: 10.13004/kjnt.2015.11.2.167

12. Kung WM, Hung KS, Chiu WT, Tsai SH, Lin JW, Wang YC, et al. Quantitative assessment of impaired postevacuation brain re-expansion in bilateral chronic subdural haematoma: Possible mechanism of the higher recurrence rate. Injury. (2012) 43:598-602. doi: 10.1016/j.injury.2010.07.240

13. Link TW, Boddu S, Marcus J, Rapoport BI, Lavi E, Knopman J. Middle meningeal artery embolization as treatment for chronic subdural hematoma: a case series. Oper Neurosurg. (2018) 14:556-62. doi: 10.1093/ons/opx154

14. Clarencon F, Blanc R, Lin C-J, Mounayer C, Galatoire O, Morax S, et al. Combined endovascular and surgical approach for the treatment of palpebral arteriovenous malformations: experience of a single center. Am J Neuroradiol. (2012) 33:148-53. doi: 10.3174/ajnr.A2735

15. Chen $\mathrm{H}$, Lin $\mathrm{X}$, Jin $\mathrm{Y}, \mathrm{Li} \mathrm{W}, \mathrm{Ma}$ G, Hu X. Patients with intralesional hemorrhage in venous malformations: diagnosis and embolosclerotherapy. $J$ Vasc Surg. (2009) 49:429-33. doi: 10.1016/j.jvs.2008.08.051

16. Paramasivam S, Toma $\mathrm{N}$, Niimi $\mathrm{Y}$, Berenstein A. De novo development of dural arteriovenous fistula after endovascular 
embolization of pial arteriovenous fistula. J Neurointerv Surg. (2013) 5:321-6. doi: 10.1136/neurintsurg-2012-010318

17. Kim E. Embolization therapy for refractory hemorrhage in patients with chronic subdural hematomas. World Neurosurg. (2017) 101:5207. doi: 10.1016/j.wneu.2017.02.070

18. Tempaku A, Yamauchi S, Ikeda H, Tsubota N, Furukawa H, Maeda D, et al. Usefulness of interventional embolization of the middle meningeal artery for recurrent chronic subdural hematoma: five cases and a review of the literature. Interv Neuroradiol. (2015) 21:366-71. doi: 10.1177/1591019915583224

19. Rimon U, Garniek A, Galili Y, Golan G, Bensaid P, Morag B. Ethanol sclerotherapy of peripheral venous malformations. Eur J Radiol. (2004) 52:283-7. doi: 10.1016/j.ejrad.2003.09.010

20. Lee C, Chen S. Direct percutaneous ethanol sclerotherapy for treatment of a recurrent venous malformation in the periorbital region. ANZ J Surg. (2004) 74:1126-7. doi: 10.1111/j.1445-1433.2004.03255.x

21. Lee C, Chen S. Direct percutaneous ethanol instillation for treatment of venous malformation in the face and neck. Br J Plast Surg. (2005) 58:10738. doi: 10.1016/j.bjps.2005.04.014

22. Prasetyono TOH, Kreshanti P. Efficacy of intra-lesional alcohol injection as alternative and/or complementary treatment of vascular malformations: a systematic review. J Plast Reconstr Aesthetic Surg. (2010) 63:10719. doi: 10.1016/j.bjps.2009.04.020

23. Cantwell C, Taubman K, Scorza L. Absolute alcohol embolization of sporadic angiomyolipoma. Semin Interv Radiol. (2008) 25:319-22. doi: 10.1055/s-0028-1085932

24. Singh P, Mishra N, Dash H, Thyalling R, Sharma B, Sarkar C, et al. Treatment of vertebral hemangiomas with absolute alcohol (ethanol) embolization, cord decompression, and single level instrumentation: a pilot study. Neurosurgery. (2011) 68:78-84. doi: 10.1227/NEU.0b013e3181fc60e9

25. Barbier C, Legeais M, Cottier JP, Bibi R, Herbreteau D. Échec De L'embolisation transartérielle par L'alcool absolu d'une fistule durale du sinus transverse. J Neuroradiol. (2008) 35:230-35. doi: 10.1016/j.neurad.2008.01.001

26. Unnikrishnan KP, Sinha PK, Sriganesh K, Suneel PR. Case report: alterations in bispectral index following absolute alcohol embolization in a patient with intracranial arteriovenous malformation. Can J Anesth. (2007) 54:90811. doi: 10.1007/BF03026795

27. Guo C, Liu L, Wang B, Wang Z. Swirl sign in traumatic acute epidural hematoma: prognostic value and surgical management. Neurol Sci. (2017) 38:2111-16. doi: 10.1007/s10072-017-3121-4

28. Peres CMA, Caldas JGMP, Puglia P, De Andrade AF, Da Silva IAF, Teixeira MJ, et al. Endovascular management of acute epidural hematomas: clinical experience with 80 cases. J Neurosurg. (2018) 128:1044-50. doi: 10.3171/2016.11.JNS161398

29. Sakai H, Takagi H, Ohtaka H, Tanabe T, Ohwada T, Yada K. Serial changes in acute extradural hematoma size and associated changes in level of consciousness and intracranial pressure. J Neurosurg. (1988) 68:56670. doi: 10.3171/jns.1988.68.4.0566

30. Knuckey N, Gelbard S, Epstein M. The management of "asymptomatic" epidural hematomas. J Neurosurg. (1989) 70:392-6. doi: 10.3171/jns.1989.70.3.0392

31. Marvin E, Laws L, Coppens J. Ruptured pseudoaneurysm of the middle meningeal artery presenting with a temporal lobe hematoma and a contralateral subdural hematoma. Surg Neurol Int. (2016) 7:S23-7. doi: 10.4103/2152-7806.173564

32. Lammy S, McConnell R, Kamel M, Rennie I, Al-Haddad S. Extradural haemorrhage: Is there a role for endovascular treatment? $\mathrm{Br} J$ Neurosurg. (2013) 27:383-5. doi: 10.3109/02688697.2012.717981

33. Takizawa K, Sorimachi T, Ishizaka H, Osada $T$, Srivatanakul K, Momose $\mathrm{H}$, et al. Enlargement of the middle meningeal artery on
MR angiography in chronic subdural hematoma. J Neurosurg. (2016) 124:1679-83. doi: 10.3171/2015.5.JNS1567

34. Ishihara $\mathrm{H}$, Ishihara $\mathrm{S}$, Kohyama $\mathrm{S}$, Yamane $\mathrm{F}$, Ogawa $M$, Sato A, et al. Experience in endovascular treatment of recurrent chronic subdural hematoma. Interv Neuroradiol. (2007) 13:1414. doi: 10.1177/15910199070130S121

35. Link TW, Rapoport BI, Paine SM, Kamel H, Knopman J. Middle meningeal artery embolization for chronic subdural hematoma: endovascular technique and radiographic findings. Interv Neuroradiol. (2018) 24:45562. doi: 10.1177/1591019918769336

36. Misaki K, Muramatsu N, Nitta H. Endovascular treatment for traumatic ear bleeding associated with acute epidural hematoma. Neurol Med Chir (Tokyo). (2008) 48:208-10. doi: 10.2176/nmc.48.208

37. Liu W, Ma L, Wen L, Shen F, Sheng H, Zhou B, et al. Drilling skull plus injection of urokinase in the treatment of epidural haematoma: a preliminary study. Brain Inj. (2008) 22:199-204. doi: 10.1080/02699050801895407

38. Nelson J. Local skull trephination before transfer is associated with favorable outcomes in cerebral herniation from epidural hematoma. Acad Emerg Med. (2011) 18:78-85. doi: 10.1111/j.1553-2712.2010.00949.x

39. Kim I, Son B, Lee S, Sung J, Hong J. Comparison of frame-based and frameless stereotactic hematoma puncture and subsequent fibrinolytic therapy for the treatment of supratentorial deep seated spontaneous intracerebral hemorrhage. Minim Invasive Neurosurg. (2007) 50:86-90. doi: 10.1055/s-2007-982503

40. Ross IB. Embolization of the middle meningeal artery for the treatment of epidural hematoma. J Neurosurg. (2009) 110:12479. doi: 10.3171/2008.7.JNS0883

41. Hashimoto T, Ohashi T, Watanabe D, Koyama S, Namatame H, Izawa $\mathrm{H}$, et al. Usefulness of embolization of the middle meningeal artery for refractory chronic subdural hematomas. Surg Neurol Int. (2013) 4:104. doi: 10.4103/2152-7806.116679

42. Link TW, Schwarz JT, Paine SM, Kamel H, Knopman J. Middle meningeal artery embolization for recurrent chronic subdural hematoma: a case series. World Neurosurg. (2018) 118:e570-4. doi: 10.1016/j.wneu.2018. 06.241

43. Yu J, Guo Y, Xu B, Xu K. Clinical importance of the middle meningeal artery : a review of the literature. Int J Med Sci. (2016) 13:790-9. doi: 10.7150/ijms. 16489

44. Hammer FD, Boon LM, Mathurin P, Vanwijck RR. Ethanol sclerotherapy of venous malformations: evaluation of systemic ethanol contamination. J Vasc Interv Radiol. (2001) 12:595-600. doi: 10.1016/S1051-0443(07)6 1482-1

45. Ernemann U, Westendorff C, Troitzsch D, Hoffmann J. Navigation-assisted sclerotherapy of orbital venolymphatic malformation: a new guidance technique for percutaneous treatment of low-flow vascular malformations. Am J Neuroradiol. (2004) 25:1792-5. Available online at: http://www.ajnr.org/ content/25/10/1792

Conflict of Interest: The authors declare that the research was conducted in the absence of any commercial or financial relationships that could be construed as a potential conflict of interest.

Copyright (C) 2020 Fan, Wang, Ding, Xu, Liu, Wang and Li. This is an open-access article distributed under the terms of the Creative Commons Attribution License (CC $B Y)$. The use, distribution or reproduction in other forums is permitted, provided the original author(s) and the copyright owner(s) are credited and that the original publication in this journal is cited, in accordance with accepted academic practice. No use, distribution or reproduction is permitted which does not comply with these terms. 